\title{
Cherry Maps with Different Critical Exponents: Bifurcation of Geometry
}

\section{B. Ndawa Tangue}

We consider order-preserving $C^{3}$ circle maps with a flat piece, irrational rotation number and critical exponents $\left(\ell_{1}, \ell_{2}\right)$.

We detect a change in the geometry of the system. For $\left(\ell_{1}, \ell_{2}\right) \in[1,2]^{2}$ the geometry is degenerate and becomes bounded for $\left(\ell_{1}, \ell_{2}\right) \in[2, \infty)^{2} \backslash\{(2,2)\}$. When the rotation number is of the form $[a b a b \ldots]$; for some $a, b \in \mathbb{N}^{*}$, the geometry is bounded for $\left(\ell_{1}, \ell_{2}\right)$ belonging above a curve defined on $] 1,+\infty\left[^{2}\right.$. As a consequence, we estimate the Hausdorff dimension of the nonwandering set $K_{f}=\mathcal{S}^{1} \backslash \bigcup_{i=0}^{\infty} f^{-i}(U)$. Precisely, the Hausdorff dimension of this set is equal to zero when the geometry is degenerate and it is strictly positive when the geometry is bounded.

Keywords: circle map, irrational rotation number, flat piece, critical exponent, geometry, Hausdorff dimension

To TIEMGNI DEFFO's family: the mother Carine and the children Abigail, Joakim, and Helza after the death of Richard, the father of the family.

Received September 01, 2020

Accepted October 27, 2020

The author was partially supported by the Centre d'Excellence Africain en Science Mathématiques et Applications (CEA-SMA).

Bertuel Ndawa Tangue

bertuelt@yahoo.fr

Institute of Mathematics and Physical Sciences

Avakpa, Porto-Novo, 613 Benin

National Advanced School of Engineering

Route Melen, Yaounde, 8390 Cameroon

RUSSIAN JOURNAL OF NONLINEAR DYNAMICS, 2020, 16(4), 651-672 


\section{Introduction}

We study a certain class of weakly order-preserving, noninjective (on an interval called a flat piece) circle maps which appear naturally in the study of Cherry flows on the two-dimensional torus (see [8, 11, 14, 15]), noninvertible continuous circle map (see [7]) and of the dependence of the rotation interval on the parameter value for one-parameter families of continuous circle maps (see [16]). The dynamics of circle maps with a flat interval has been intensively explored in the past years, see $[4,5,7,14,17,18]$.

We discuss the geometry of the nonwandering set (a set obtained by removing from the circle all preimages of the flat piece). Where the geometry is concerned, we discover a dichotomy which generalizes the one found in [5]. Some of our maps show a "degenerate geometry", while others seem to be subject to the "bounded geometry".

Before we can explain more precisely our results, we introduce our class, adopt some notations and present basic lemmas.

\subsection{The class of functions}

We fix $\ell_{1}, \ell_{2} \geqslant 1$ and consider the class $\mathscr{L}$ of continuous circle maps $f$ of degree one for which an $\operatorname{arc} U$ exists so that the following properties hold:

1. The image of $U$ is one point.

2. The restriction of $f$ to $\mathcal{S}^{1} \backslash \bar{U}$ is a $C^{3}$-diffeomorphism onto its image.

3. Let $(a, b)$ be a preimage of $U$ under the projection of the real line to $\mathcal{S}^{1}$. In some right-sided neighborhood of $b, f$ can be represented as

$$
h_{r}\left((x-b)^{\ell_{2}}\right),
$$

where $h_{r}$ is a $C^{3}$-diffeomorphism in a two-sided neighborhood of $b$. Analogously, in a leftsided neighborhood of $a, f$ equals

$$
h_{l}\left((x-a)^{\ell_{1}}\right) .
$$

In the following, we assume that $h_{l}(x)=h_{r}(x)=x$. In fact, it is possible to make $C^{3}$ coordinate changes near $a$ and $b$ that will allow us to replace both $h_{l}$ and $h_{r}$ by the identity function.

Let $F$ be a lift of $f$ on the real line. The rotation number $\rho(f)$ of $f$ is defined (independently of $x$ and $F$ ) by

$$
\rho(f):=\lim _{n \rightarrow \infty} \frac{F^{n}(x)-x}{n}(\bmod 1) .
$$

Let $\left(q_{n}\right)$ be the sequence of denominators of the convergents of $\rho(f)$ (irrational) defined recursively by $q_{1}=1, q_{2}=a_{1}$ and $q_{n+1}=a_{n} q_{n}+q_{n-1}$ for all $n \geqslant 3$, with

$$
\rho(f)=\left[a_{0} a_{1} \ldots\right]:=a_{0}+\frac{1}{a_{1}+\frac{1}{a_{2}+\frac{1}{\ddots}}}
$$




\section{Additional Assumption}

Let $f \in \mathscr{L}$. We say that $S f$ (the Schwarzian derivative of $f$ ) is negative if

$$
S f(x):=\frac{D^{3} f(x)}{D f(x)}-\frac{3}{2}\left(\frac{D^{2} f(x)}{D f(x)}\right)^{2}<0, \quad \forall x, D f(x) \neq 0,
$$

with $D^{n} f$ being the $n^{\text {th }}$ derivative of $f$, for $n \in \mathbb{N}$.

We will assume in the proof of the first part of Theorem 1 that $f$ has a negative negative Schwarzian derivative.

\subsection{Notations and Definitions}

The fundamental notations are established in [5, pp. 2-3]. Let $f \in \mathscr{L}$.

1. For every $i \in \mathbb{Z}$, the symbol $\underline{i}$ means $f^{i}(U)$.

2. Let $I$ and $J$ be two intervals. $(I, J)$ is the interval between $I$ and $J .[I, J):=I \cup(I, J)$ and $(I, J]:=(I, J) \cup J .|I|$ is the length of the interval $I,|[I, J)|:=|I|+|(I, J)|$ and $|(I, J]|:=|J|+|(I, J)|$. We say that $I$ and $J$ are comparable when $|I|$ and $|J|$ are comparable. This means that there is $k>0$ such that $\frac{1}{k}|I|<|J|<k|I|$.

3. For any sequence $\Gamma_{n}$ and for any real $d$, we adopt the following notation:

$$
\Gamma_{n}^{d\left(\ell_{1}, \ell_{2}\right)}:=\left\{\begin{array}{ll}
\Gamma_{n}^{d \ell_{1}} & \text { if } n \equiv 0[2], \\
\Gamma_{n}^{d \ell_{2}} & \text { if } n \equiv 1[2],
\end{array} \quad \Gamma_{n}^{d\left(\frac{1}{\ell_{1}}, \frac{1}{\ell_{2}}\right)}:=\left\{\begin{array}{l}
\Gamma_{n}^{d \frac{1}{\ell_{1}}} \text { if } n \equiv 0[2] \\
\Gamma_{n}^{\frac{1}{\ell_{2}}} \text { if } n \equiv 1[2] .
\end{array}\right.\right.
$$

\subsection{Discussion and statement of the results}

\section{Scaling ratios}

The sequence

$$
\alpha_{n}:=\frac{\left.\mid \underline{\left(-q_{n}\right.}, \underline{0}\right) \mid}{\left.\mid \underline{-q_{n}}, \underline{0}\right) \mid}=\frac{\left|\left(f^{-q_{n}}(U), U\right)\right|}{\left|\left(f^{-q_{n}}(U), U\right)\right|+\left|f^{-q_{n}}(U)\right|}
$$

measures the geometry near a critical point. In fact, it serves as scaling relating the geometries of successive dynamical partitions.

The geometry is said to be degenerate when $\alpha_{n}$ goes to zero and bounded when $\alpha_{n}$ is bounded away from zero.

The study of this geometry is parameterized by a rotation number and critical exponents. In [5], for pairs $(\ell, \ell) ; \ell>1$ and $\rho$ the irrational number of bounded type (i.e., $\max _{n} a_{n}<\infty$ ), the authors found a transition between degenerate geometry and bounded geometry. In fact, they show under (A1) that, if $1<\ell \leqslant 2$ and $\rho \in \mathbb{R} \backslash \mathbb{Q}$, then the geometry is degenerate and it is bounded (independently of (A1)) if $\ell>2$ and $\rho$ is an irrational number of bounded type. In [4], for $\ell>1$, the author proved that the class of function $f$ of critical exponents $(1, \ell)$ or $(\ell, 1)$ has a degenerate geometry. In [15], the authors show that, for the maps in $\mathscr{L}$ with Fibonacci rotation number, when the critical exponents $\left(\ell_{1}, \ell_{2}\right)$ belong to $(1,2)^{2}$, the geometry is degenerate. Let us note that, in contrast to other previous works, information on the geometry of the system is obtained by the study of the asymptotic behavior of the renormalization operator.

RUSSIAN JOURNAL OF NONLINEAR DYNAMICS, 2020, 16(4), 651-672 
In the present paper, we consider the cases where the critical exponents $\left(\ell_{1}, \ell_{2}\right)$ belong to a subdomain (containing the previous domains) of $[1, \infty)^{2}$; also, the rotation number is not necessarily of Fibonacci type and the results do not depend on the renormalization operator as in [9]. We use the formalism presented in [5] which is based on recursive inequalities analysis of $\alpha_{n}$. For technical reasons, in the case of bounded geometry, we introduce the vector sequence $v_{n}:=\left(-\ln \alpha_{n},-\ln \alpha_{n-1}\right)$ and, the new recursive inequality is controlled by a $2 \times 2$ matrix. When the rotation number is bi-periodic $(\rho=[a b a b \ldots] ; a, b \in \mathbb{N})$, the $2 \times 2$ matrix has two eigenvalues (depending on the rotation number and critical exponents) $\lambda_{s} \in(0,1)$ and $\lambda_{u}>0$. The equation $\lambda_{u}=\lambda_{u}\left((a, b) ;\left(\ell_{1}, \ell_{2}\right)\right)=1$ defines a curve $\mathcal{C}_{\lambda_{u}=1}$ (presented above) which separates the $\left(\ell_{1}, \ell_{2}\right)$ plan into two components $C_{\lambda_{u}>1}$ (below the curve) and $C_{\lambda_{u}<1}$.

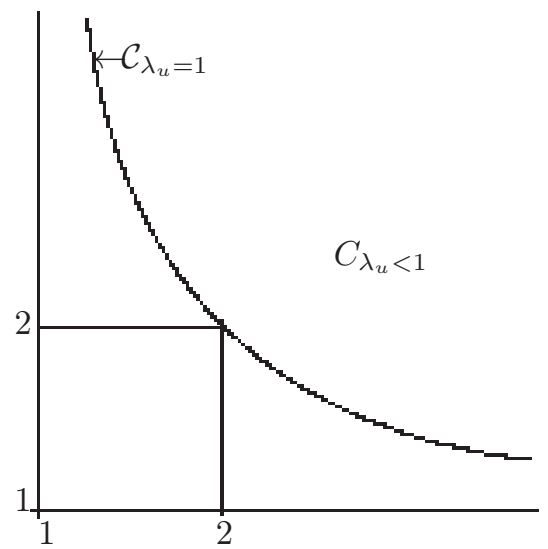

1. Main result Let $f \in \mathscr{L}$ with critical exponents $\left(\ell_{1}, \ell_{2}\right)$. Then

$1)$ the scaling ratio $\alpha_{n}$ goes to zero when $\left(\ell_{1}, \ell_{2}\right) \in[1,2]^{2}, \rho \in \mathbb{R} \backslash \mathbb{Q}$ and (A1) holds;

$2)$ the scaling ratio $\alpha_{n}$ is bounded away from zero when $\left(\ell_{1}, \ell_{2}\right) \in[2, \infty)^{2} \backslash\{(2,2)\}$ and $\rho$ is of bounded type;

3) the scaling ratio $\alpha_{n}$ is bounded away from zero when $\left(\ell_{1}, \ell_{2}\right) \in C_{\lambda_{u}<1}$ and $\rho$ is bi-periodic.

2. Estimation of Hausdorff dimension of the nonwandering set. In the symmetric case $\left(\ell_{1}=\ell_{2}=\ell\right)$, in [8], for $f \in \mathscr{L}$ with a rotation number of bounded type, the author shows that, if $\ell \in(1,2]$, then the Hausdorff dimension of the nonwandering set is equal to zero, and that, if $\ell>2$, then the Hausdorff dimension of the nonwandering set is strictly greater than zero. This result generalizes the one in [19] where the author treats the maps in $\mathscr{L}$ with critical exponents $(1,1)$. Note that the results in [8] are more general (they only depend on geometry); precisely, if the rotation number is of bounded type, then the Hausdorff dimension of the nonwandering set is equal to zero when the geometry is degenerate and it is strictly greater than zero when the geometry is bounded; so we have the following result which is proved at the end of the paper.

Corollary. Let $f \in \mathscr{L}$ with critical exponent $\left(\ell_{1}, \ell_{2}\right)$ with a rotation number of bounded type. Then

1) the Hausdorff dimension of the nonwandering set is equal to zero when $\left(\ell_{1}, \ell_{2}\right) \in[1,2]^{2}$ and (A1) holds and for the pairs $(\ell, 1) ; \ell>1$;

2) the Hausdorff dimension is strictly larger than zero when $\left(\ell_{1}, \ell_{2}\right) \in[2, \infty)^{2} \backslash\{(2,2)\}$;

3) the Hausdorff dimension is strictly larger than zero when $\left(\ell_{1}, \ell_{2}\right) \in C_{\lambda_{u}<1}$ and $\rho$ is biperiodic. 
The following remark will simplify the statements and proof of the results.

REMARK 1. Note that our setting has some inherent symmetry. This will simplify the statements and proof of our results.

\section{Tools}

\subsection{Cross-ratio inequalities}

Notation 1. We denote by $\mathbb{R}_{<}^{4}$ the subset of $\mathbb{R}^{4}$ defined by

$$
\mathbb{R}_{<}^{4}:=\left\{\left(x_{1}, x_{2}, x_{3}, x_{4}\right) \in \mathbb{R}^{4}, \text { such that } x_{1}<x_{2}<x_{3}<x_{4}\right\} .
$$

The following result can be found in [1] (Theorem 2)

Proposition 1. The cross-ratio inequality $(\boldsymbol{C R I})$.

Let $f \in \mathscr{L}$. Let $(a, b, c, d) \in \mathbb{R}_{<}^{4}$. The cross-ratio $C r$ is defined by

$$
\operatorname{Cr}(a, b, c, d):=\frac{|b-a||d-c|}{|c-a||d-b|}
$$

and the cross-ratio Poin is defined by

$$
\operatorname{Poin}(a, b, c, d):=\frac{|d-a||b-c|}{|c-a||d-b|}
$$

The distortion of the cross-ratio $\mathrm{Cr}$ and cross-ratio Poin are given, respectively, by

$$
\mathcal{D} C r(a, b, c, d):=\frac{\operatorname{Cr}(f(a), f(b), f(c), f(d))}{\operatorname{Cr}(a, b, c, d)}
$$

and

$$
\mathcal{D P o i n}(a, b, c, d):=\frac{\operatorname{Poin}(f(a), f(b), f(c), f(d))}{\operatorname{Poin}(a, b, c, d)} .
$$

Let us consider a set of $n+1$ quadruples $\left\{a_{i}, b_{i}, c_{i}, d_{i}\right\}$ with the following properties:

1) each point of the circle belongs to at most $k$ intervals $\left(a_{i}, d_{i}\right)$;

2) the intervals $\left(b_{i}, c_{i}\right)$ do not intersect $U$.

Then there is $C_{k}, C_{k}^{\prime}>0$ such that the following inequalities hold:

$$
\prod_{i=0}^{n} \mathcal{D} \operatorname{Cr}\left(a_{i}, b_{i}, c_{i}, d_{i}\right) \leqslant C_{k}
$$

and

$$
\prod_{i=0}^{n} \mathcal{D} \boldsymbol{P o i n}\left(a_{i}, b_{i}, c_{i}, d_{i}\right) \geqslant C_{k}^{\prime} .
$$

Observe that Poin $+\mathbf{C r}=1$. Thus, by Lemma 1.5 in [2] we have the following result. 
Proposition 2. Let $f$ be a $C^{3}$ function such that the Schwarzian derivative is negative. Then $C_{k}^{\prime}>1$, that is, $C_{k}<1$.

REMARK 2. Let $I$ and $J$ be two intervals of finite and nonzero length such that $\bar{I} \cap \bar{J}=\varnothing$. We assume that $J$ is on the right of $I$ and we put $I:=[a, b]$ and $J:=[c, d]$, then

$$
\operatorname{Cr}(I, J):=\frac{|I||J|}{|[I, J)||(I, J]|}=\mathbf{C r}(a, b, c, d)
$$

and

$$
\text { Poin }(I, J):=\frac{|(I, J)||[I, J]|}{|[I, J)||(I, J]|}=\text { Poin }(a, b, c, d) \text {. }
$$

Fact 1. Let $f \in \mathscr{L}$. Let $l(U)$ and $r(U)$ be the left and right endpoints of $U$ (the flat piece of $f)$, respectively. There are a left-sided neighborhood $I^{l}$ of $l(U)$, a right-sided neighborhood $I^{r}$ of $r(U)$ and three positive constants $K_{1}, K_{2}, K_{3}$ such that the following holds:

1. If $y \in I^{l_{i}}$ with $l_{1}:=l, l_{2}:=r$, then

$$
\begin{gathered}
K_{1}\left|l_{i}(U)-y\right|^{l_{i}} \leqslant\left|f\left(l_{i}(U)\right)-f(y)\right| \leqslant K_{2}\left|l_{i}(U)-y\right|^{l_{i}}, \\
K_{1}\left|l_{i}(U)-y\right|^{l_{i}-1} \leqslant \frac{d f}{d x}(y) \leqslant K_{2}\left|l_{i}(U)-y\right|^{l_{i}-1} .
\end{gathered}
$$

2. If $y \in(x, z) \subset I^{l_{i}}$, with $z$ the closest point to the flat interval $U$, then

$$
\frac{|f(x)-f(y)|}{|f(x)-f(z)|} \leqslant K_{3} \frac{|x-y|}{|x-z|}
$$

The first part of Fact 1 implies that

$$
f_{\mid I^{l_{i}}} \approx k_{i} x^{\ell_{i}}, i=1,2 \text {. We assume that } \quad k_{1}=k_{2} .
$$

We need this assumption to prove that $\alpha_{n}$ goes to zero (Lemma 1 and Lemma 2).

\subsection{Basic Results}

Proposition 3. Let $n \geqslant 1$.

- The set of "long" intervals consists of the intervals

$$
\mathcal{A}_{n}:=\left\{\left(\underline{i}, \underline{q_{n}+i}\right) ; 0 \leqslant i \leqslant q_{n+1}-1\right\} .
$$

- The set of "short" intervals consists of the intervals

$$
\mathcal{B}_{n}:=\left\{\left(\underline{q_{n+1}+i}, \underline{i}\right) ; 0 \leqslant i \leqslant q_{n}-1\right\} .
$$

The set $\mathcal{P}_{n}:=\mathcal{A}_{n} \cup \mathcal{B}_{n}$ covers the circle modulo the end points and the flat piece and it is called the nth dynamical partition.

The dynamical partition produced by the first $\underline{q_{n+1}+q_{n}}$ preimages of $U$ is denoted by $\mathcal{P}^{n}$. It consists of

$$
\wp_{n}:=\left\{\underline{-i} ; 0 \leqslant i \leqslant q_{n+1}+q_{n}-1\right\}
$$


together with the gaps between these sets. As in the case of $\mathcal{P}_{n}$, there are two kinds of gaps, "long" and "short":

- The set of "long" intervals consists of the intervals

$$
\mathcal{A}^{n}:=\left\{\left(\underline{-q_{n}-i}, \underline{-i}\right)=: I_{i}^{n} ; 0 \leqslant i \leqslant q_{n+1}-1\right\} .
$$

- The set of "short" intervals consists of the intervals

$$
\mathcal{B}^{n}:=\left\{\left(\underline{-i}, \underline{-q_{n+1}-i}\right)=: I_{i}^{n+1} ; 0 \leqslant i \leqslant q_{n}-1\right\} .
$$

Proposition 4. The sequence $\left|\left(\underline{0}, \underline{q_{n}}\right)\right|$ tends to zero at least exponentially fast.

Proposition 5. If $A$ is a preimage of $U$ belonging to $\mathcal{P}^{n}$ and if $B$ is one of the gaps adjacent to $A$, then $|A| /|B|$ is bounded away from zero by a constant that does not depend on $n, A$ or $B$.

Lemma 1. The sequence

$$
f\left(\sigma_{n}\right)=\frac{\left|\left(\underline{1}, \underline{q_{n}+1}\right)\right|}{\left.\mid \underline{q_{n-1}+1}, \underline{1}\right) \mid}
$$

is bounded.

The proofs of these results can be found in [5] (proof of Proposition 1, Proposition 2 and Lemma 1.3).

A proof of the following proposition can be found in [3, Theorem 3.1, p. 285].

Proposition 6 (Koebe principle). Let $f \in \mathscr{L}$. For every $\varsigma, \alpha>0$, there exists a constant $\zeta(\varsigma, \alpha)>0$ such that the following holds. Let $T$ and $M \subset T$ be two intervals and let $S, D$ be the left and the right component of $T \backslash M$ and $n \in \mathbb{N}$. Suppose that:

1) $\sum_{i=0}^{n-1} f^{i}(T)<\varsigma$,

2) $f^{n}: T \longrightarrow f^{n}(T)$ is a diffeomorphism,

3) $\frac{\left|f^{n}(M)\right|}{\left|f^{n}(S)\right|}, \frac{\left|f^{n}(M)\right|}{\left|f^{n}(D)\right|}<\alpha$.

Then

$$
\frac{1}{\zeta(\varsigma, \alpha)} \leqslant \frac{D f^{n}(x)}{D f^{n}(y)} \leqslant \zeta(\varsigma, \alpha), \quad \forall x, y \in M
$$

that is,

$$
\frac{1}{\zeta(\varsigma, \alpha)} \cdot \frac{|A|}{|B|} \leqslant \frac{f^{n}(A)}{f^{n}(B)} \leqslant \zeta(\varsigma, \alpha) \cdot \frac{|A|}{|B|}, \quad \forall A, B \text { (intervals) } \subseteq M
$$

where,

$$
\zeta(\varsigma, \alpha)=\frac{1+\alpha}{\alpha} e^{C \varsigma}
$$

and $C \geqslant 0$ only depends on $f$.

Remark 3. Let $f \in \mathscr{L}$. Given $n>1, T$ and $M$ as before, $f^{n}: T \longrightarrow f^{n}(T)$ is a diffeomorphism if and only if, for all $0 \leqslant i \leqslant n-1, f^{i}(T) \cap \bar{U}=\varnothing$, where $\bar{U}$ denotes the closure of $U$. 


\section{Proof of results}

Let us put together the (parameter) sequences which are frequently used in this section.

$$
\alpha_{n}=\frac{\left|\left(\underline{-q_{n}}, \underline{0}\right)\right|}{\left.\mid \underline{-q_{n}}, \underline{0}\right) \mid}, \quad \sigma_{n}=\frac{\left|\left(\underline{0}, \underline{q_{n}}\right)\right|}{\left|\underline{q_{n-1}}, \underline{0}\right|}, \quad s_{n}:=\frac{\left|\left[\underline{-q_{n-2}}, \underline{0}\right]\right|}{|\underline{0}|}, \quad \tau_{n}:=\frac{\left|\left(\underline{0}, \underline{q_{n}}\right)\right|}{\left|\left(\underline{0}, \underline{q_{n-2}}\right)\right|}
$$

and

$$
\beta_{n}(k)=\frac{\left.\mid \underline{\left(-q_{n}+k q_{n-1}\right.}, \underline{0}\right) \mid}{\left.\mid \underline{\underline{-q_{n}+k q_{n-1}}}, \underline{0}\right) \mid}, \quad k=0,1, \ldots a_{n-1} .
$$

\subsection{Proof of the first part of the main result}

\subsubsection{A priori bounds of $\alpha_{n}$}

Proposition 7. Let $n \in \mathbb{N}$ and $\left(\ell_{1}, \ell_{2}\right) \in \Omega_{0}=[1,2]^{2}$.

For all $\alpha_{n}$,

$$
\alpha_{n}^{\frac{\ell_{1}, \ell_{2}}{2}}<0.55
$$

for at least every other $\alpha_{n}$

$$
\alpha_{n}^{\frac{\ell_{1}, \ell_{2}}{2}}<0.3
$$

If

$$
\alpha_{n}^{\frac{\ell_{1}, \ell_{2}}{2}}>0.3
$$

then either

$$
\alpha_{n}^{\frac{\ell_{1}, \ell_{2}}{2}}<0.44 \text { or } \quad \alpha_{n+1}^{\frac{\ell_{1}, \ell_{2}}{2}}<0.16 \text {. }
$$

Proof. For every $n \in \mathbb{N}$ and $k=0,1, \ldots a_{n-1}$, we define the parameter sequences

$$
\begin{gathered}
\gamma_{1, n}(k):=\left|\left(\underline{-q_{n}+k q_{n-1}}, \underline{0}\right)\right|, \quad \gamma_{1, n-1}:=\gamma_{1, n-1}(0), \quad \gamma_{n}(k)=\frac{\gamma_{1, n}(k)}{\gamma_{1, n-1}} \\
\gamma_{n}^{\left(\ell_{1} \mid \ell_{2}\right)}(k):=\frac{\gamma_{1, n}^{\ell_{1}}(k)}{\gamma_{1, n-1}^{\ell_{2}}} \text { if } n \in 2 \mathbb{Z} \text { and } \gamma_{n}^{\left(\ell_{1} \mid \ell_{2}\right)}(k):=\frac{\gamma_{1, n}^{\ell_{2}}(k)}{\gamma_{1, n-1}^{\ell_{1}}} \text { if } n \in 2 \mathbb{Z}+1 .
\end{gathered}
$$

These notations simplify the formalization of the following lemma which will play an important (essential) role in the proof of Proposition 7.

Lemma 2. For $n$ large enough and for every $k=0,1, \ldots a_{n-1}-1$, the following inequality holds:

$$
\frac{\left(\beta_{n}(k)^{\ell_{1}, \ell_{2}}+\alpha_{n-1}^{\ell_{1}, \ell_{2}} \gamma_{n}^{\left(\ell_{1} \mid \ell_{2}\right)}(k)\right)\left(1+\gamma_{n}^{\left(\ell_{1} \mid \ell_{2}\right)}(k)\right)}{\left(1+\alpha_{n-1}^{\ell_{1}, \ell_{2}} \gamma_{n}^{\left(\ell_{1} \mid \ell_{2}\right)}(k)\right)\left(\beta_{n}(k)^{\ell_{1}, \ell_{2}}+\gamma_{n}^{\left(\ell_{1} \mid \ell_{2}\right)}(k)\right)} \leqslant s_{n} \beta_{n}(k+1) .
$$

Proof. Let $n$ be an even nonnegative large enough integer. For fixed $k=0,1, \ldots a_{n-1}-1$, according to assumption (A2), the left-hand side of (3.1) is equal to the cross-ratio

$$
\text { Poin }\left(\underline{-q_{n}+k q_{n-1}+1}, \underline{-q_{n-1}+1}\right) \text {. }
$$

Applying $f^{q_{n-1}-1}$, by expanding the cross-ratio property, we get the inequality. 
The left-hand side is a function of the three variables $\beta_{n}(k)^{\ell_{1}, \ell_{2}}, \alpha_{n-1}^{\ell_{1}, \ell_{2}}$ and $\gamma_{n}^{\left(\ell_{1} \mid \ell_{2}\right)}$. Observe that the function increases monotonically with each of the first two variables. However, with respect to the third variable, the function reaches a minimum. To see this, take the logarithm of the function and check that the first derivative is equal to zero only when

$$
\left(\gamma_{n}^{\left(\ell_{1} \mid \ell_{2}\right)}\right)^{2}=\frac{\beta_{n}(k)^{\ell_{1}, \ell_{2}}}{\alpha_{n-1}^{\ell_{1}, \ell_{2}}}
$$

By substituting this value for $\gamma_{n}^{\left(\ell_{1} \mid \ell_{2}\right)}$ in (3.1), we get

$$
\left(\frac{\beta_{n}(k)^{\frac{\ell_{1}, \ell_{2}}{2}}+\alpha_{n-1}^{\frac{\ell_{1}, \ell_{2}}{2}}}{1+\beta_{n}(k)^{\frac{\ell_{1}, \ell_{2}}{2}} \alpha_{n-1}^{\frac{\ell_{1}, \ell_{2}}{2}}}\right)^{2} \leqslant s_{n} \beta_{n}(k+1) .
$$

Put:

$$
x_{n}(k):=\min \left\{\beta_{n}(k)^{\frac{\ell_{1}, \ell_{2}}{2}}, \alpha_{n-1}^{\frac{\ell_{1}, \ell_{2}}{2}}\right\} \quad \text { and } \quad y_{n}(k):=\beta_{n}(k)^{\frac{\ell_{1}, \ell_{2}}{2}} .
$$

Since $\beta_{n}(k+1) \leqslant y_{n}(k+1)$, substituting the above variable into (3.2) gives rise to a quadratic inequality in $x_{n}(k)$ whose only root in the interval $(0,1)$ is given by

$$
\frac{\sqrt{s_{n} y_{n}(k+1)}}{1+\sqrt{1-s_{n} y_{n}(k+1)}}
$$

that is,

$$
x_{n}(k)=\min \left\{\beta_{n}(k)^{\frac{\ell_{1}, \ell_{2}}{2}}, \alpha_{n-1}^{\frac{\ell_{1}, \ell_{2}}{2}}\right\} \leqslant \frac{\sqrt{s_{n} y_{n}(k+1)}}{1+\sqrt{1-s_{n} y_{n}(k+1)}} .
$$

Lemma 3. There is a subsequence of $\alpha_{n}$ including at least every other $\alpha_{n}$, such that

$$
\lim \sup \alpha_{n}^{\frac{\ell_{1}, \ell_{2}}{2}} \leqslant 0.3
$$

Proof. We use the following elementary lemma in [5].

Lemma 4. The function

$$
h_{n}(z)=\frac{\sqrt{s_{n} z}}{1+\sqrt{1-s_{n} z}}
$$

moves points to the left, $h(z)<z$, if $z \geqslant 0.3$ and $n$ is large enough.

We select the subsequence.

1. The initial term: there exists $n-2 \in \mathbb{N}$ such that $\frac{\frac{\ell_{1}, \ell_{2}}{2}}{\alpha_{n-2}} \leqslant 0.3$. This comes directly from the properties of the function $h_{n}$ (Lemma 4) and from (3.3).

2. The next element: suppose that $\alpha_{n-2}$ has been selected. If

$$
x_{n}(k)=\alpha_{n-1}^{\frac{\ell_{1}, \ell_{2}}{2}} \quad \text { or } \quad \alpha_{n-1}^{\frac{\ell_{1}, \ell_{2}}{2}} \leqslant 0.3
$$

for some $k=0,1, \ldots a_{n-1}-1$, then we select $\alpha_{n-1}$ as the next term. Otherwise, $\alpha_{n}$ is the next term. Thus, the sequence is constructed. 
Corollary 1. For the whole sequence $\left(\alpha_{n}\right)$ we have

$$
\lim \sup \alpha_{n}^{\frac{\ell_{1}, \ell_{2}}{2}} \leqslant 0.3
$$

Moreover,

if $\alpha_{n-1}$ does not belong to the subsequence $\left(\alpha_{n}\right)$ defined by Lemma 3 , then either

$$
\alpha_{n-1}^{\frac{\ell_{1}, \ell_{2}}{2}}<0.44 \quad \text { or } \quad \alpha_{n}^{\frac{\ell_{1}, \ell_{2}}{2}}<0.16 \text {. }
$$

Proof. Observe that the function

$$
H:(s, t) \in \mathbb{R}_{+}{ }^{2} \mapsto F(s, t)=\frac{s+t}{1+s t}
$$

is symmetric and, for fixed $s$, the function $F(s, \cdot)$ reaches its minimum in zero by taking the value $s$. Therefore, for every $s, t \geqslant 0$,

$$
s, t \leqslant \frac{s+t}{1+s t}
$$

So,

$$
\alpha_{n}^{\frac{\ell_{1}, \ell_{2}}{2}}, \alpha_{n-1}^{\frac{\ell_{1}, \ell_{2}}{2}} \leqslant \frac{\alpha_{n}^{\frac{\ell_{1}, \ell_{2}}{2}}+\alpha_{n-1}^{\frac{\ell_{1}, \ell_{2}}{2}}}{1+\alpha_{n}^{\frac{\ell_{1}, \ell_{2}}{2}} \alpha_{n-1}^{\frac{\ell_{1}, \ell_{2}}{2}}} .
$$

Hence, $\alpha_{n-2}$ is an element of the sequence and suppose that $\alpha_{n-1}$ do not belong to the previous subsequence of Lemma 3, then it follows from (3.2) that the right member of (3.4) is estimated as follows:

$$
\frac{\alpha_{n}^{\frac{\ell_{1}, \ell_{2}}{2}}+\alpha_{n-1}^{\frac{\ell_{1}, \ell_{2}}{2}}}{1+\alpha_{n}^{\frac{\ell_{1}, \ell_{2}}{2}} \alpha_{n-1}^{\frac{\ell_{1}, \ell_{2}}{2}}} \leqslant \sqrt{s_{n} \beta_{n}(1)^{\frac{\ell_{1}, \ell_{2}}{2}}} \approx \sqrt{\beta_{n}(1)^{\frac{\ell_{1}, \ell_{2}}{2}}} \leqslant \sqrt{0.3} .
$$

Also,

$$
\min \left\{\alpha_{n}^{\frac{\ell_{1}, \ell_{2}}{2}}, \alpha_{n-1}^{\frac{\ell_{1}, \ell_{2}}{2}}\right\}=\alpha_{n}^{\frac{\ell_{1}, \ell_{2}}{2}} \leqslant 0.3
$$

Thus, if $\frac{\ell_{n}, \ell_{2}}{2} \geqslant 0.16$, then by combining this with (3.5) and (3.6), we obtain the desired estimate.

Proposition 7 is proved.

\subsubsection{Recursive formula of $\alpha_{n}$}

Proposition 8. Let $n$ be a large enough integer,

1) if $\ell_{1}, \ell_{2}>1$, we have

$$
\alpha_{2 n}^{\ell_{1}} \leqslant M_{2 n}\left(\ell_{1}\right) \alpha_{2 n-2}^{2} \quad \text { and } \quad \alpha_{2 n+1}^{\ell_{2}} \leqslant M_{2 n+1}\left(\ell_{2}\right) \alpha_{2 n-1}^{2},
$$

where

$$
M_{n}(\ell)=s_{n-1}^{2} \cdot \frac{2}{\ell} \cdot \frac{1}{1+\sqrt{1-\frac{2(\ell-1)}{\ell} s_{n-1} \alpha_{n-1}}} \cdot \frac{1}{1-\alpha_{n-2}} \cdot \frac{\sigma_{n}}{\sigma_{n-2}}
$$

2) if $\ell_{1}=\ell_{2}=1$, then

$$
\alpha_{n} \leqslant W_{n}^{1} \cdot \frac{\sigma_{n}}{\sigma_{n-2}} \alpha_{n-2}
$$


Proof. We treat the case $n$ even and the case $n$ odd is treated in a similar way. Recall that

$$
\alpha_{n}=\frac{\left|\left(\underline{-q_{n}}, \underline{0}\right)\right|}{\left.\mid \underline{-q_{n}}, \underline{0}\right) \mid} .
$$

For all even $n$ large enough, by Proposition 4 and point 2 of Fact 1, applying $f$ to the equality, we get

$$
\alpha_{n}^{\ell_{1}}=\frac{\left|\left(\underline{-q_{n}+1}, \underline{1}\right)\right|}{\left.\mid \underline{-q_{n}+1}, \underline{1}\right) \mid}
$$

which is certainly less than the cross-ratio

$$
\operatorname{Poin}\left(\underline{-q_{n}+1},\left(\underline{1}, \underline{-q_{n-1}+1}\right]\right) .
$$

Since the cross-ratio Poin is expanded by $f^{q_{n-1}-1}$, then

$$
\alpha_{n}^{\ell_{1}}<\delta_{n}(1) s_{n}(1)
$$

with

$$
\delta_{n}(k):=\frac{\left|\left(\underline{-q_{n}+k q_{n-1}}, \underline{k q_{n-1}}\right)\right|}{\left.\mid \underline{\underline{-q_{n}+k q_{n-1}}}, \underline{k q_{n-1}}\right) \mid}
$$

and

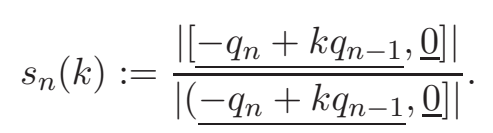

If $a_{n-1}=1$, by multiplying and dividing the right member of (3.9) by $\alpha_{n-2}^{2}$, we obtain directly (3.11).

Suppose that $a_{n-1}>1$ and estimate $\delta_{n}(k)$. By the mean value (Lagrange) theorem, $f$ transforms the intervals defining the ratio $\delta_{n}(k)$ into a pair whose ratio is

$$
\frac{u_{k}}{v_{k}} \delta_{n}(k)
$$

with $u_{k}$ being the derivative of $f\left(x^{\ell_{1}}\right)$ at a point in the interval

$$
\left.U_{k}:=\underline{\left(-q_{n}+k q_{n-1}\right.}, \underline{k q_{n-1}}\right),
$$

and $v_{k}$ being the derivative of $f\left(x^{\ell_{1}}\right)$ at a point in the interval

$$
V_{k}:=\left[\underline{-q_{n}+k q_{n-1}}, \underline{k q_{n-1}}\right) .
$$

Note that, for $n$ sufficiently large,

$$
u_{1}<v_{1}<u_{2}<v_{2} \ldots<v_{a_{n-1}} .
$$

We see that the image of $\delta_{n}(k)$ by $f$ is smaller than

$$
\operatorname{Poin}\left(\underline{-q_{n}+k q_{n-1}+1}, \underline{\left(k q_{n-1}+1\right.}, \underline{-q_{n-1}+1}\right) \text {. }
$$

Once again, by expanding the cross-ratio property $\left(f^{q_{n-1}-1}\right)$, it follows that

$$
\frac{u_{k}}{v_{k}} \delta_{n}(k) \leqslant s_{n}(k+1) \cdot \delta_{n}(k+1) .
$$


Multiplying (3.10) for $k=0, \ldots, a_{n-1}-1$, and substituting the resulting estimate of $\delta_{n}(1)$ into (3.9), we obtain

$$
\alpha_{n}^{\ell_{1}} \leqslant \delta_{n}\left(a_{n-1}\right) \frac{v_{a_{n-1}}}{u_{1}} s_{n}(1) \ldots s_{n}\left(a_{n-1}\right) .
$$

Observe that $s_{n}(1) \ldots s_{n}\left(a_{n-1}\right)<s_{n}$ and

$$
\frac{v_{a_{n-1}}}{u_{1}} \leqslant\left(\frac{\left|\left(\underline{-q_{n-2}}, \underline{0}\right)\right|}{\mid \underline{\left(\underline{q_{n-1}}, \underline{0}\right) \mid}}\right)^{\ell_{1}-1} \leqslant \frac{\left|\left(\underline{-q_{n-2},}, \underline{0}\right)\right|}{\left|\left(\underline{q_{n-1}}, \underline{0}\right)\right|} .
$$

Thus,

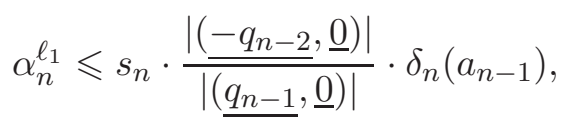

which can be rewritten as

$$
\alpha_{n}^{\ell_{1}} \leqslant s_{n} \nu_{n-2} \mu_{n-2} \alpha_{n-2}^{2}
$$

with

$$
\nu_{n-2}:=\frac{\left|\left[\underline{-q_{n-2}}, \underline{0}\right)\right|}{\left|\underline{\left(\underline{q_{n-1}}, \underline{0}\right)}\right|} \cdot \frac{\left|\left[\underline{-q_{n-2}}, \underline{0}\right)\right|}{\left.\mid \underline{\left[-q_{n-2}\right.}, \underline{a_{n-1} q_{n-1}}\right) \mid}
$$

and

$$
\mu_{n-2}:=\frac{\mid\left(\underline{-q_{n-2}}, \underline{\left.a_{n-1} q_{n-1}\right) \mid}\right.}{\left.\mid \underline{\left(-q_{n-2}\right.}, \underline{0}\right) \mid} .
$$

It remains to estimate $\nu_{n-2}$ and $\mu_{n-2}$ to complete this part. For $\nu_{n-2}$, observe that

$$
\left|\left(\underline{-q_{n-2}}, \underline{0}\right)\right| \leqslant\left|\left(\underline{q_{n-3}}, \underline{0}\right)\right|,
$$

so that

$$
\nu_{n-2} \leqslant \frac{1}{\sigma_{n-1} \sigma_{n-2}} \cdot \frac{1}{1-\alpha_{n-2}} .
$$

The estimation of $\mu_{n-2}$ is facilitated by the following elementary lemma in [5].

Lemma 5. Let $\ell \in(1,2)$. For all numbers $x>y$, we have the following inequality:

$$
\frac{x^{\ell}-y^{\ell}}{x^{\ell}} \geqslant\left(\frac{x-y}{x}\right)\left[\ell-\frac{\ell(\ell-1)}{2}\left(\frac{x-y}{x}\right)\right] .
$$

Now apply $f$ into the intervals defining the ratio $\mu_{n-2}$. By Lemma 5 , the resulting ratio is larger than

$$
\mu_{n-2}\left(\ell_{1}-\frac{\ell_{1}\left(\ell_{1}-1\right)}{2} \mu_{n-2}\right) .
$$

The cross-ratio Poin

$$
\text { Poin }\left(-q_{n-2}+1,\left(\underline{q_{n-1}+1}, \underline{1}\right)\right) \text {, }
$$

that is,

$$
\frac{\left|\underline{\left(-q_{n-2}+1\right.}, \underline{\left.q_{n-1}+1\right)}\right|\left|\left[\underline{\underline{-q_{n-2}+1}}, \underline{1}\right)\right|}{\left.\left|\underline{\left[-q_{n-2}+1\right.}, \underline{\left.q_{n-1}+1\right)}\right| \mid \underline{\left(-q_{n-2}+1\right.}, \underline{1}\right) \mid}
$$


is larger again. Thus, by expanding the cross-ratio property on $f^{q_{n-2}}$, we obtain

$$
\mu_{n-2}\left(\ell_{1}-\frac{\ell_{1}\left(\ell_{1}-1\right)}{2} \mu_{n-2}\right) \leqslant s_{n-1} \sigma_{n} \sigma_{n-1} .
$$

By solving this quadratic inequality, we obtain

$$
\mu_{n-2} \geqslant \frac{1+\sqrt{1-\frac{2}{\ell_{1}}\left(\ell_{1}-1\right) s_{n-1} \sigma_{n} \sigma_{n-1}}}{\ell_{1}-1} .
$$

Thus, by combining (3.13) and (3.14), we obtain

$$
\mu_{n-2}<\frac{2}{\ell_{1}} \cdot \frac{1}{1+\sqrt{1-\frac{2\left(\ell_{1}-1\right)}{\ell_{1}} s_{n-1} \sigma_{n} \sigma_{n-1}}} s_{n-1} \sigma_{n} \sigma_{n-1} .
$$

Since $\sigma_{n} \sigma_{n-1}<\alpha_{n-1}$, the first inequality in (3.7) follows by combining inequalities (3.11), (3.12) and (3.15). Likewise, the second inequality in (3.7) is obtained by following suitably the same reasoning as previously.

\subsection{3. $\alpha_{n}$ go to zero}

If $\ell_{1}=\ell_{2}=1$, then by Proposition $7, \prod_{k=2}^{k=n} W_{k}^{1}$ goes to zero; thus, by composing the inequality obtained by $f$, since $f\left(\sigma_{n}\right)$ is bounded (Lemma 1 ), the result follows.

Note that the cases where the critical exponents are of the form $(1, \ell)$ or $(\ell, 1)$, with $\ell>1$, are treated in [4].

Now let us suppose that $\ell_{1}, \ell_{2}>1$.

Technical reformulation of Proposition 8.

Let $W_{n}$ be a sequence defined by

$$
M_{n}(\ell)=W_{n}(\ell) \frac{\sigma_{n}}{\sigma_{n-2}} .
$$

Let

$$
M_{n}^{\prime}(\ell):=M_{n}(\ell) \alpha_{n-2}^{2-\ell} \quad \text { and } \quad W_{n}^{\prime}(\ell):=W_{n}(\ell) \alpha_{n-2}^{2-\ell} .
$$

The recursive formula (3.7) can be written for $n$ even in the form

$$
\alpha_{n}^{\ell_{1}} \leqslant W_{n}^{\prime}\left(\ell_{1}\right) \frac{\sigma_{n}}{\sigma_{n-2}} \alpha_{n-2}^{\ell_{1}} .
$$

So,

$$
\alpha_{n}^{\ell_{1}} \leqslant \prod_{k=2}^{k=n} W_{k}^{\prime}\left(\ell_{1}\right) \frac{\sigma_{n}}{\sigma_{0}} \alpha_{0}^{\ell_{1}}
$$

$\prod_{k=2}^{k=n} W_{k}^{\prime}\left(\ell_{1}\right)$ goes to zero.

Observe that the size of $W_{n}^{\prime}\left(\ell_{1}\right)$ is given by the study of the function

$$
W_{n}^{\prime}\left(x, y, \ell_{1}\right)=\frac{1}{\frac{\ell_{1}}{2}+\frac{\ell_{1}}{2} \sqrt{1-\frac{2\left(\ell_{1}-1\right)}{\ell_{1}} x^{\frac{2}{\ell_{2}}}}} \cdot \frac{y^{\frac{4}{\ell_{1}}-2}}{1-y^{\frac{2}{\ell_{1}}}} .
$$

The meaning of the variation of $W_{n}^{\prime}\left(x, y, \ell_{1}\right)$ relative to the third variable is given by the following lemma in [5]. 
Lemma 6. For any $0<y<\frac{1}{\sqrt{e}}, x \in(0,1)$ and $\ell_{1} \in(1,2]$ the function $W_{n}^{\prime}\left(x, y, \ell_{1}\right)$ is increasing with respect to $\ell_{1}$.

\section{Analysis of the asymptotic size of $W_{i}^{\prime}(2)$}

Since the hypotheses of Lemma 6 are satisfied (Proposition 7), the only remaining point is the verification of the convergence of $\prod_{i=1}^{n} W_{i}^{\prime}(2)$.

- If $\alpha_{n-2}<(0.3)^{\ell_{1}}$, then $W^{\prime}(2)<W^{\prime}(0.55,0.16,2)<0,9$.

- If not, then by Proposition $7, W^{\prime}(2)<W^{\prime}(0.3,0.44,2)<0,98$ or else, $W_{n+1}^{\prime}(2) W_{n}^{\prime}(2)<$ $W^{\prime}(0.55,0.16,2) W^{\prime}(0.16,0.55,2)<0,85$.

Corollary 2. Let $\ell_{1}, \ell_{2} \in[1,2]$. If $1<\ell_{1}<2$, respectively, $1<\ell_{2}<2$, then $\alpha_{2 n}$, respectively, $\alpha_{2 n+1}$ goes to zero least double exponentially fast. And if $\ell_{1}=2$ or 1 , respectively, $\ell_{2}=2$ or 1 , then $\alpha_{2 n}$, respectively, $\alpha_{2 n+1}$ goes to zero least exponentially fast

Proof. Let $n:=2 p_{n} \in \mathbb{N}$. From the analysis of the asymptotic size of $W_{i}^{\prime}(2)$, it follows that, as $n$ goes to infinity, $\prod_{i=0}^{n} W_{i}^{\prime}\left(\ell_{1}\right)$ goes to zero and so does $\alpha_{n}$. Therefore,

$$
\prod_{i=0}^{n} M_{i}\left(\ell_{1}\right)
$$

goes to zero as $n$ goes to infinity. Thus, by Proposition 8 , for $n$ even, there is $\lambda_{0}$ such that

- if $1<\ell_{1}<2$,

$$
\alpha_{n} \leqslant \lambda_{0}^{\left(\frac{2}{\ell_{1}}\right)^{p_{n}}}
$$

- and if $\ell_{1}=1,2$,

$$
\alpha_{n} \leqslant \lambda_{0}^{n}
$$

The case $n$ odd is treated in the same way.

\subsection{Proof of the second part of the main result}

In this section we find a bounded geometry domain.

\subsubsection{Recursive affine inequality of order two on $\alpha_{n}$}

Let

$$
\kappa_{n}:=\frac{\left|\left(\underline{0}, \underline{q_{n}}\right)\right|}{\left|\left(\underline{0}, \underline{-q_{n-1}}\right)\right|} .
$$

Remark 4. Since the point $\underline{q_{n-2}}$ lies in the gap between $\underline{-q_{n-1}}$ and $-q_{n-1}+q_{n-2}$ of the dynamical partition $\mathcal{P}_{n-2}$, it follows by Proposition 5 that $\tau_{n} / \alpha_{n-1}$ and $\frac{-q_{n}-1}{\kappa_{n}}$ are comparable.

Proposition 9. For any rotation number of bounded type, there is a uniform constant $K$ such that

$$
\kappa_{2 n}>K\left(\alpha_{2 n-1}\right) \frac{1-\ell_{2}^{a_{2 n}+1}}{\ell_{2}-1} \quad \text { and } \quad \kappa_{2 n+1}>K\left(\alpha_{2 n}\right) \frac{1-\ell_{1}^{a_{2 n+1}+1}}{\ell_{1}-1} \text {. }
$$


1. Proof of the proposition: If $a_{n}=1$, it comes down to showing that the sequence $\kappa_{n}$ is bounded away from zero, which becomes relatively very simple. In fact, suppose that $\left|\left(\underline{q_{n}}, \underline{-q_{n-1}}\right)\right| \leqslant\left|\left(\underline{0}, \underline{q_{n}}\right)\right|$, then

$$
\kappa_{n}=\frac{\left|\left(\underline{0}, \underline{q_{n}}\right)\right|}{\left|\left(\underline{0}, \underline{-q_{n-1}}\right)\right|} \geqslant \frac{1}{2},
$$

else, $\kappa_{n}$ is greater than

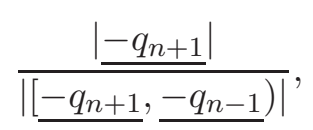

which by Proposition 5 is bounded away from zero.

In the following part of the proof, we suppose that $a_{n}>1$ and the following lemma in [5] (Lemma 4.1) is used.

Lemma 7 . The ratio

$$
1-\beta_{n}(i)=\frac{\left|-q_{n}+i q_{n-1}\right|}{\left.\mid \underline{\left.-q_{n}+i q_{n-1}\right)}, \underline{0}\right) \mid}
$$

is bounded away from zero by a uniform constant for all $i=0, \ldots, a_{n-1}$.

Lemma 8. For every $n \in \mathbb{N}$ and for all $i=0, \ldots, a_{n-1}$, there is a uniform constant $K$ such that

$$
\beta_{n}(i)^{\ell_{1}, \ell_{2}} \geqslant \beta_{n}(i+1) .
$$

Proof. For $n$ large enough and for fixed $i=0, \ldots, a_{n-1}$, by Proposition 4 and Fact 1 , we have

which is greater than

$$
\beta_{n}(i)^{\ell_{1}, \ell_{2}}=\frac{\left|\left(\underline{-q_{n}+k q_{n-1}+1}, \underline{1}\right)\right|}{\left.\mid \underline{-q_{n}+i q_{n-1}+1}, \underline{1}\right) \mid},
$$

$$
\operatorname{Cr}\left(\left[\underline{-q_{n-2}+1}, \underline{-q_{n}+i q_{n-1}+1}\right),\left(\underline{-q_{n}+i q_{n-1}+1}, \underline{1}\right)\right) .
$$

By applying the cross-ratio inequality under $f^{q_{n-2}-1}$, by Fact 1 the resulting ratio is greater than

$$
\operatorname{Cr}\left(\frac{\left[-q_{n-2}+1\right.}{\left.\left(\underline{-q_{n}+i q_{n-1}+q_{n-2}+1}, q_{n-2}+\underline{1}\right)\right)},\right.
$$

times a uniform constant. We now repeat this sequence of steps $a_{n-2}-1$ times: Apply $f^{q_{n-2}-1}$, discard the interval containing $\underline{0}$ and use point 2 of Fact 1 , and replace the result by a cross-ratio spanning the intervals $-q_{n-2}+1$. At the end, this will produce the cross ratio

$$
\begin{aligned}
& \operatorname{Cr}\left(\left[\underline{-q_{n-2}+1}, \underline{-q_{n}+i q_{n-1}+a_{n-2} q_{n-2}+1}\right),\right. \\
& \left.\left(\underline{-q_{n}+i q_{n-1}+a_{n-2} q_{n-2}+1}, a_{n-2} q_{n-2}+\underline{1}\right)\right) \text {. }
\end{aligned}
$$

And finally, by applying $f^{q_{n-3}-1}$, since by Lemma 7 the interval containing $-q_{n-2}+q_{n-3}$ is bounded away from zero, the resulting ratio is

$$
\frac{\left.\mid \underline{\left(-q_{n}+(i+1) q_{n-1}\right.}, \underline{q_{n-1}}\right) \mid}{\left.\mid \underline{\left[-q_{n}+(i+1) q_{n-1}\right.}, \underline{q_{n-1}}\right) \mid}
$$

times a uniform constant. Thus, since $\underline{-q_{n}+q_{n-1}}$ lies between $\underline{-q_{n}+(i+1) q_{n-1}}$ and $\underline{q_{n-1}}$, it follows by Lemma 7 that this ratio is comparable to $\beta_{n}(i+1)$. 
2. Back to the proof of Proposition 9: Observe by Proposition 5 that

$$
\left.\mid \underline{\left(-q_{n}+(i+1) q_{n-1}\right.}, \underline{0}\right) \mid
$$

and

$$
\left.\mid \underline{\left[-q_{n}+i q_{n-1}\right.}, \underline{0}\right) \mid
$$

are comparable. Therefore, $\kappa_{n-1}$ is comparable to the product

$$
\beta_{n}(1) \ldots \beta_{n}\left(a_{n-1}-1\right) .
$$

By combining this with Lemma 8, we have Proposition 9.

\section{Recursive affine inequality of order two on $\alpha_{n}$.}

Proposition 10. If $\rho(f)$ is of bounded type, then there is a uniform constant $K$ such that

$$
\alpha_{2 n} \geqslant K\left(\alpha_{2 n-1}\right)^{\frac{\ell_{2}}{\ell_{1}}} \cdot \frac{1-\ell_{2}^{-a_{2 n}}}{\ell_{2}-1}\left(\alpha_{2 n-2}\right)^{\ell_{1}^{-a_{2 n-1}}}
$$

and

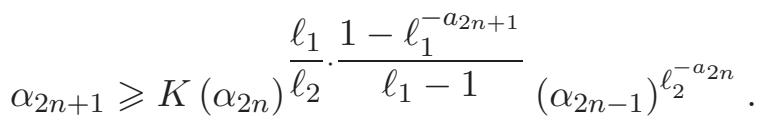

4. Proof of the proposition. If $n$ is even and large enough, then

$$
\alpha_{n}^{\ell_{1}}=\frac{\left.\mid \underline{\left(-q_{n}+1\right.}, \underline{1}\right) \mid}{\left.\mid \underline{-q_{n}+1}, \underline{1}\right) \mid},
$$

which in turn is larger than the product of two ratios

$$
\xi_{1, n}=\frac{\left|\left(\underline{-q_{n}+1}, \underline{1}\right)\right|}{\left|\underline{\left(-q_{n}+1,\right.} \underline{\left.-q_{n-1}+1\right)}\right|} \quad \text { and } \quad \xi_{2, n}=\frac{\mid \underline{\left(\underline{-q_{n}+1}, \underline{-q_{n-1}+1}\right) \mid}}{\left.\mid \underline{\underline{-q_{n}+1}}, \underline{-q_{n-1}+1}\right) \mid} \text {. }
$$

Lemma 9. For all even $n$ large enough

$$
\xi_{1, n} \geqslant K \tau_{n}
$$

Proof. Observe that $\xi_{1, n}$ is greater than

$$
\operatorname{Cr}\left(\left(\underline{-q_{n}+1}, \underline{1}\right), \underline{\left.-q_{n-1}+1\right)}\right) \text {. }
$$

By applying CRI on $f^{q_{n-1}-1}$ and discarding the intervals containing $\underline{0}$, repeat this $a_{n-1}-1$ times more: By Fact 1, the resulting ratio is larger than

$$
\operatorname{Cr}\left(\left(\underline{\left(-q_{n-2}-q_{n-1}+1\right.}, \underline{\left.\left(a_{n-1}-1\right) q_{n-1}+1\right)}\right),\left(\underline{1}, \underline{\left.-q_{n-1}+1\right]}\right)\right) .
$$

Apply $f^{q_{n-1}-1}$ and discard the intervals containing the flat interval. Apply $f$, replace the resulting by the cross-ratio

$$
\left.\operatorname{Cr}\left(\left(\underline{-q_{n-2}+1}, \underline{q_{n-1}+1}\right),\left(\underline{1}, \underline{-q_{n-1}+1}\right]\right)\right) .
$$


Thus, by CRI on $f^{q_{n-2}-1}$ and the inequalities above, we obtain

$$
\xi_{1, n}>\frac{\left.\mid \underline{\left(q_{n-2},-q_{n-3}\right.}\right] \mid}{\left.\mid \underline{q_{n}}, \underline{-q_{n-3}}\right) \mid} \tau_{n} .
$$

The first factor on the right-hand side of (3.16) is greater than

$$
\frac{\mid \underline{-q_{n-3} \mid}}{\left|\left(\underline{0}, \underline{-q_{n-3}}\right)\right|},
$$

which by Proposition 5 goes away from zero. The lemma is proved.

Lemma 10. There is a uniform constant $K$ so that, for all $n$,

$$
\xi_{2,2 n} \geqslant K\left(\alpha_{2 n-2}\right)^{\ell_{1}^{-a_{2 n-1}+1}} \quad \text { and } \quad \xi_{2,2 n+1} \geqslant K\left(\alpha_{2 n-1}\right)^{\ell_{1}^{-a_{2 n}+1}} .
$$

Proof. If $a_{n-1}=1$, then $\xi_{2, n}$ is greater than

$$
\operatorname{Cr}\left(\left[\underline{-q_{n-2}+1}, \underline{-q_{n}+1}\right),\left(\underline{-q_{n}+1}, \underline{-q_{n-1}+1}\right)\right) .
$$

By applying CRI $\left(q_{n-2}-1\right)$, the resulting ratio is greater than

$$
\operatorname{Cr}\left(\left[\underline{-q_{n-2}+1}, \underline{-q_{n-1}+1}\right),\left(\underline{-q_{n-1}+1}, \underline{-q_{n-3}+1}\right)\right)
$$

times a uniform constant. Thus, by applying CRI to this ratio with $f^{q_{n-3}-1}$, the above inequalities and Proposition 5, the result follows.

Now suppose that $a_{n-1}>1$, then $\xi_{2, n}$ is greater than

$$
\operatorname{Cr}\left(\left[\underline{-q_{n}+q_{n-1}+1}, \underline{-q_{n}+1}\right),\left(\underline{-q_{n}+1}, \underline{-q_{n-1}+1}\right)\right) .
$$

By applying $f^{q_{n-1}-1}$ to this ratio, it follows from Lemma 7 that

$$
\xi_{2, n} \geqslant K^{\prime} \beta_{n}(1) \text {. }
$$

And the lemma follows from this by using Lemma 8 modulo the fact that

$$
\beta_{n}\left(a_{n-1}\right)=\alpha_{n-2} \text {. }
$$

Combining Lemma 9, Lemma 10, Proposition 9 and Remark 4, the result of Proposition 10 follows.

REMARK 5. By the inequality obtained from Proposition 10, the sequence $\nu_{n}$ defined by

$$
\nu_{n}=-\ln \alpha_{n}
$$

verifies the following recursive affine inequalities of order two for every $n>0$

$$
\nu_{2 n} \leqslant \frac{\ell_{2}}{\ell_{1}} \cdot t_{2}\left(a_{2 n}\right) \nu_{2 n-1}+\ell_{1}^{-a_{2 n-1}} \nu_{2 n-2}+\widetilde{K^{\prime}}
$$

and

$$
\nu_{2 n+1} \leqslant \frac{\ell_{1}}{\ell_{2}} \cdot t_{1}\left(a_{2 n+1}\right) \nu_{2 n}+\ell_{2}^{-a_{2 n}} \nu_{2 n-1}+\widetilde{K^{\prime}}
$$

with

$$
t_{i}(j)=\frac{1-\ell_{i}^{-j}}{\ell_{i}-1}
$$




\subsubsection{Analysis of the recursive affine inequality}

We will prove that the sequence $\nu_{n}$ is bounded. Let us consider the sequence of vectors $\left(v_{n}\right)$ defined by

$$
v_{n}=\left(\begin{array}{c}
\nu_{n} \\
\nu_{n-1}
\end{array}\right)
$$

the vector given by

$$
\bar{\kappa}=\left(\begin{array}{c}
\widetilde{K^{\prime}} \\
0
\end{array}\right)
$$

and the sequence matrix

$$
A_{\ell_{1}, \ell_{2}}(2 n)=\left(\begin{array}{cc}
\frac{\ell_{2}}{\ell_{1}} \cdot t_{2}\left(a_{2 n}\right) & \ell_{1}^{-a_{2 n-1}} \\
1 & 0
\end{array}\right)
$$

and

$$
A_{\ell_{1}, \ell_{2}}(2 n+1)=\left(\begin{array}{cc}
\frac{\ell_{1}}{\ell_{2}} \cdot t_{1}\left(a_{2 n+1}\right) & \ell_{2}^{-a_{2 n}} \\
1 & 0
\end{array}\right),
$$

say the associated matrix to the recursive affine tnequalities (3.17) and (3.18), respectively, in the sense that (3.17) and (3.18) can be rewritten, respectively, as

$$
v_{2 n} \leqslant A_{\ell_{1}, \ell_{2}}(2 n) v_{2 n-1}+\bar{\kappa} \quad \text { and } \quad v_{2 n+1} \leqslant A_{\ell_{2}, \ell_{1}}(2 n+1) v_{2 n}+\bar{\kappa} .
$$

Therefore, for every $n:=2 p_{n}+r_{n}$, with $p_{n} \in \mathbb{N}^{*}$ and $r_{n} \in\{0,1\}$, we have

$$
\begin{aligned}
v_{n} \leqslant & \bar{A}_{\ell_{1}, \ell_{2}}(n) \bar{A}_{\ell_{1}, \ell_{2}}(n-2) \ldots \bar{A}_{\ell_{1}, \ell_{2}}\left(2+r_{n}\right) v_{2-r_{n}}+ \\
& \left(I d+\sum_{i=4+r_{n}}^{n} \bar{A}_{\ell_{1}, \ell_{2}}(n) \bar{A}_{\ell_{1}, \ell_{2}}(n-2) \ldots \bar{A}_{\ell_{1}, \ell_{2}}(i)\right) \bar{\kappa}^{\prime},
\end{aligned}
$$

where

$$
\bar{A}_{\ell_{1}, \ell_{2}}(n)=A_{\ell_{1}, \ell_{2}}(n) A_{\ell_{1}, \ell_{2}}(n-1) .
$$

Observe that, if $\left(\ell_{1}, \ell_{2}\right)$ is very close to an element of the set

$$
\{(a, \infty),(\infty, b),(\infty, \infty), a, b \in \mathbb{R}\},
$$

$\bar{A}_{\ell_{1}, \ell_{2}}(n)$ is diagonalizable with nonnegative eigenvalues, at most one of them being strictly positive, that is, $1 / \ell_{1}$ or $1 / \ell_{2}$ and as $\ell_{1}, \ell_{2}>2$, then $\bar{A}_{\ell_{1}, \ell_{2}}(n)$ contracts the Euclidean metric; therefore, $v_{n}$ (also $\nu_{n}$ and $\alpha_{n}$ ) is bounded. In the following analysis, we suppose that $1<\ell_{1}$, $\ell_{2}<C<\infty$ for some $C$ in $\mathbb{R}_{+}$.

Lemma 11. Fix $\left(\ell_{1}, \ell_{2}\right) \in[2, \infty)^{2}$. The sequence

$$
\bar{A}_{\ell_{1}, \ell_{2}}^{\circ n}:=\bar{A}_{\ell_{1}, \ell_{2}}(n) \bar{A}_{\ell_{1}, \ell_{2}}(n-2) \ldots \bar{A}_{\ell_{1}, \ell_{2}}(4)
$$

is bounded (uniformly) by $\max \left\{\ell_{1} / \ell_{2}, \ell_{2} / \ell_{1}\right\}$. 
Proof. Observe that, for all $n \in \mathbb{N}$,

$$
\bar{A}_{\ell_{1}, \ell_{2}}(n) \leqslant\left(\begin{array}{cc}
\left(1-b_{n}(2)\right)\left(1-b_{n-1}(2)\right) & \frac{\ell_{1}}{\ell_{2}}\left(1-b_{n}(2)\right) b_{n-2}(2) \\
\frac{\ell_{2}}{\ell_{1}}\left(1-b_{n-1}(2)\right) & b_{n-2}(2)
\end{array}\right)=: \bar{B}_{\ell_{1}, \ell_{2}}(n)
$$

with

$$
b_{2 n}(\ell)=b_{2 n}:=\ell_{2}^{-a_{2 n}} \quad \text { and } \quad b_{2 n+1}(\ell)=b_{2 n+1}:=\ell_{1}^{-a_{2 n+1}} .
$$

Let be a sequence $\left(x_{n}\right)_{n \in \mathbb{N}}$ defined by

$$
\left(x_{n}\right)_{n \in \mathbb{N}}:=\left\{1-b_{n}(2), b_{n}(2) ; n \in \mathbb{N}\right\} .
$$

Remark that, for every $n \in \mathbb{N}, x_{n} \in\left[2^{-a}, 1-2^{-a}\right]$, where, $a:=\max \left\{a_{n}, n \in \mathbb{N}\right\}$.

Thus, by setting

$$
\bar{B}_{\ell_{1}, \ell_{2}}^{\circ n}=\left(\begin{array}{cc}
d^{1, n} & \frac{\ell_{2}}{\ell_{1}} d^{3, n} \\
\frac{\ell_{1}}{\ell_{2}} d^{2, n} & d^{4, n}
\end{array}\right)
$$

it follows that, for every $i \in\{1,2,3,4\}$, there is $x_{k_{i, j}}, j=p_{n-2}, \ldots, n-2$ such that

$$
d^{i, n} \leqslant \sum_{j=p_{n-2}}^{n-2} x_{k_{i, 1}} \ldots x_{k_{i, j}} \leqslant 1
$$

This proves the lemma.

Proposition 11. If $\left(\ell_{1}, \ell_{2}\right) \in[2, \infty)^{2} \backslash\{(2,2)\}$, then $\bar{A}_{\ell_{1}, \ell_{2}}^{\circ n}$ contracts the Euclidean metric provided $n$ is large enough. The scale of the contraction is bounded away from 1 independently of $\ell_{1}$ and $\ell_{2}$ and the particular sequence $b_{n}$, whereas the moment when the contraction starts depends on the upper bound of $b_{n}$.

Proof. For fixed $n \in \mathbb{N}$, putting

$$
\bar{A}_{\ell_{1}, \ell_{2}}^{\circ n}=\left(\begin{array}{cc}
d^{1, n}\left(z_{1}, z_{2}\right) & \frac{\ell_{1}}{\ell_{2}} d^{3, n}\left(z_{1}, z_{2}\right) \\
\frac{\ell_{2}}{\ell_{1}} d^{2, n}\left(z_{1}, z_{2}\right) & d^{4, n}\left(z_{1}, z_{2}\right)
\end{array}\right)
$$

with $z_{1}=1 /\left(\ell_{1}-1\right)$ and $z_{2}=1 /\left(\ell_{2}-1\right)$, we find that $d^{i, n}\left(z_{1}, z_{2}\right), i=1,2,3,4$ are polynomials of respective degree $n-2, n-3, n-3$ and $n-4$, whose coefficients belong to the interval $\left[\ell^{-a}, 1-\ell^{-a}\right]$, with $\ell=\max \left\{\ell_{1}, \ell_{2}\right\}$. For fixed $i \in\{1,2,3,4\}$, we denote by $d_{j}^{i, n}$ the coefficients of $d^{i, n}\left(z_{1}, z_{2}\right)$. Set

$$
\left\{\begin{array}{lll}
d_{1}(2)=1 \quad \text { and } \quad d_{1}(i)=0 ; & i=1,3,4 \\
d_{2}(3)=1 & \text { and } \quad d_{2}(i)=0 ; & i=1,2,4
\end{array}\right.
$$

Then by Lemma 11, the sums

$$
\sum_{j=0}^{p_{n-2}} d^{i, j} z_{1}^{j+d_{1}(i)} z_{2}^{j+d_{2}(i)} ; \quad i=1,2,3,4
$$


are uniformly bounded. Therefore, for every $i \in\{1,2,3,4\}$

$$
\sum_{j=k}^{\infty} d_{j}^{i, n} z_{1}^{j+d_{1}(i)} z_{2}^{j+d_{2}(i)} \longrightarrow 0 \quad \text { as } k \longrightarrow \infty
$$

Lemma 12. For every $i \in\{1,2,3,4\}$, the sequence $\left(d_{j}^{i, n}\right)$ tends to zero at least exponentially.

Proof. By a simple calculation, we have

$$
d^{1, n}(0,0)=\prod_{i=1}^{p_{n-2}} b_{n-2 i+1}, d^{4, n}(0,0)=\prod_{i=1}^{p_{n-2}} b_{n-2 i}, d^{2, n}(0,0)=d^{3, n}(0,0)=0 .
$$

Now suppose that, for given $0<n-1$ and $0<j<n-1$, all the coefficients $d_{j}^{i, n-1} i \in\{1,2,3,4\}$ tend to zero at least exponentially fast. Then, since

$$
\bar{A}_{\ell_{1}, \ell_{2}}^{\circ n}=\bar{A}_{\ell_{1}, \ell_{2}}(n) \bar{A}_{\ell_{1}, \ell_{2}}^{\circ n-1}
$$

and by the form of the coefficients of $\bar{A}_{\ell_{1}, \ell_{2}}(n)$, the lemma and hence Proposition 11 is proved.

\subsubsection{Particular case of Bounded Geometry}

Proposition 12. Let $f \in \mathscr{L}$ with critical exponents $\left(\ell_{1}, \ell_{2}\right)$ and rotation number $\rho(f)=$ $=[a b a b \ldots]$ for some $a, b \in \mathbb{R}$. If the inequality

$$
\begin{array}{r}
\sqrt{\left(\ell_{1}^{-b}-\ell_{2}^{-a}\right)^{2}+\left(t_{1}(b) t_{2}(a)+2\left(\ell_{1}^{-b}+\ell_{2}^{-a}\right)\right) t_{1}(b) t_{2}(a)}+ \\
+t_{1}(b) t_{2}(a)+\ell_{1}^{-b}+\ell_{2}^{-a}-2<0
\end{array}
$$

holds, then the geometry of $f$ is bounded.

Proof. If $\rho(f)=[a b a b \ldots]$, then

$$
\bar{A}_{\ell_{1}, \ell_{2}}=\left(\begin{array}{cc}
t_{1}(b) t_{2}(a)+\ell_{1}^{-b} & \frac{\ell_{2}}{\ell_{1}} \ell_{2}^{-a} t_{2}(a) \\
\frac{\ell_{1}}{\ell_{2}} t_{1}(b) & \ell_{2}^{-a}
\end{array}\right)
$$

and the eigenvalues $\lambda_{s}$ and $\lambda_{u}$ are defined as follows:

$$
\begin{array}{r}
2 \lambda_{s}=-\sqrt{\left(\ell_{1}^{-b}-\ell_{2}^{-a}\right)^{2}+\left(t_{1}(b) t_{2}(a)+2\left(\ell_{1}^{-b}+\ell_{2}^{-a}\right)\right) t_{1}(b) t_{2}(a)}+ \\
+t_{1}(b) t_{2}(a)+\ell_{1}^{-b}+\ell_{2}^{-a}
\end{array}
$$

and

$$
\begin{aligned}
& 2 \lambda_{u}=\sqrt{\left(\ell_{1}^{-b}-\ell_{2}^{-a}\right)^{2}+\left(t_{1}(b) t_{2}(a)+2\left(\ell_{1}^{-b}+\ell_{2}^{-a}\right)\right) t_{1}(b) t_{2}(a)}+ \\
& +t_{1}(b) t_{2}(a)+\ell_{1}^{-b}+\ell_{2}^{-a} \text {. }
\end{aligned}
$$

Observe that $\lambda_{s} \in(0,1)$. Thus, if $\lambda_{u}<1$, then $\bar{A}_{\ell_{1}, \ell_{2}}$ contracts the Euclidean metric.

This proves the proposition. 


\subsection{Proof of the Corollary}

Lemma 13. Let

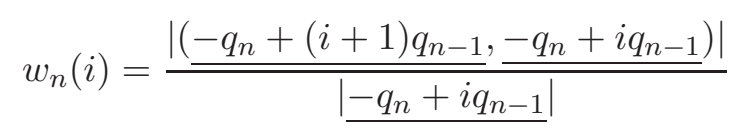

be a parameter sequence with $i=0 \ldots a_{n-1}-1 . w_{n}(i), i=1 \ldots a_{n-1}-1$ and $w_{n}^{\ell_{1}, \ell_{2}}(0)$ are comparable to $\alpha_{n-1}$.

Proof. Suppose that $a_{n-1}>1$ and let $i=1, \ldots, a_{n-1}-1$. We apply Proposition 6 to

- $\left.T=\underline{\left[-q_{n}+(i+1) q_{n-1}\right.}, \underline{-q_{n}+(i-1) q_{n-1}}\right]$,

- $M=\left(\underline{-q_{n}+(i+1) q_{n-1}}, \underline{-q_{n}+(i-1) q_{n-1}}\right)$,

- $S=\underline{-q_{n}+(i+1) q_{n-1}}$,

- $D=\underline{-q_{n}+(i-1) q_{n-1}}$,

- $f^{q_{n}-(i-1) q_{n-1}}$.

1) For every $j<q_{n}-(i-1) q_{n-1}, f^{j}(T) \cap \bar{U}=\varnothing$; so $f^{q_{n}-(i-1) q_{n-1}}$ is a diffeomorphism on $T$ (Remark 3);

2) the set $\bigcup_{j=0}^{q_{n}-(i-1) q_{n-1}}(T)$ covers the circle at most two times;

3) for $n$ large enough, by Proposition 5, we have

$$
\frac{f^{q_{n}-(i-1) q_{n-1}}(M)}{f^{q_{n}-(i-1) q_{n-1}(S)}}<\frac{f^{q_{n}-(i-1) q_{n-1}}(M)}{f^{q_{n}-(i-1) q_{n-1}(D)}}=\frac{\left|\left(\underline{0},-2 q_{n-1}\right)\right|}{\underline{\left|-2 q_{n-1}\right|}}<K .
$$

Therefore, it follows from Proposition 6 and Proposition $5\left(\left|\underline{\mid-q_{n-1}}\right|\right.$ and $\left|\left(\underline{0}, \underline{-q_{n-1}}\right]\right|$ are comparable) that $w_{n}(i)$ and $\alpha_{n-1}$ are comparable.

For $i=0$ (which is the only case where $a=1$ ), we apply Proposition 6 to

- $\left.T=\underline{\left[-q_{n}+q_{n-1}+1\right.}, \underline{-q_{n}-q_{n-1}+1}\right]$,

- $M=\left(\underline{-q_{n}+q_{n-1}+1}, \underline{-q_{n}-q_{n-1}+1}\right)$,

- $S=\underline{-q_{n}+q_{n-1}+1}$,

- $D=\underline{-q_{n}-q_{n-1}+1}$,

- $f^{q_{n}-q_{n-1-1}}$.

As before, the hypotheses are satisfied. And for $n$ large enough,

$$
w_{n}^{\ell_{1}, \ell_{2}}(0)=\frac{\left|\left(-q_{n}+q_{n-1}+1,-q_{n}+1\right)\right|}{\mid \underline{\left|-q_{n}+1\right|}},
$$

which is also uniformly comparable to $\alpha_{n-1}$.

This concludes the proof.

The rest of the proof of the corollary is as in [8] (Theorem 1.4 and Theorem 1.5). 


\section{Acknowledgments}

I sincerely thank Prof. M. Martens and Prof. Dr. L. Palmisano for introducing me to the subject of this paper, for valuable advice, continuous encouragement and helpful discussions. I thank Prof. Dr. Carlos Ogouyandjou for participating in the presentations related to this work.

\section{Conflict of Interest}

The author declares that he has no conflict of interest.

\section{References}

[1] de Melo, W. and van Strien, S., One-Dimensional Dynamics: The Schwarzian Derivative and Beyond, Bull. Amer. Math. Soc. (N.S.), 1988, vol. 18, no. 2, pp.159-162.

[2] de Melo, W. and van Strien, S., A Structure Theorem in One-Dimensional Dynamics, Ann. of Math. (2), 1989, vol. 129, no. 3, pp. 519-546.

[3] de Melo, W. and van Strien, S., One-Dimensional Dynamics, Ergeb. Math. Grenzgeb. (3), vol. 25, Berlin: Springer, 1993.

[4] Graczyk, J., Dynamics of Circle Maps with Flat Spots, Fund. Math., 2010, vol. 209, no. 3, pp. 267-290.

[5] Graczyk, J., Jonker, L.B., Świątek, G., Tangerman, F. M., and Veerman, J. J. P., Differentiable Circle Maps with a Flat Interval, Commun. Math. Phys., 1995, vol. 173, no. 3, pp. 599-622.

[6] Mendes, P., A Metric Property of Cherry Vector Fields on the Torus, J. Differential Equations, 1991, vol. 89, no. 2, pp. 305-316.

[7] Martens, M. and Palmisano, L., Invariant Manifolds for Non-Differentiable Operators, arXiv:1704.06328 (2017).

[8] Martens, M., Strien, S., Melo, W., and Mendes, P., On Cherry Flows, Ergodic Theory Dynam. Systems, 1990, vol. 10, no. 3, pp. 531-554.

[9] Misiurewicz, M., Rotation Interval for a Class of Maps of the Real Line into Itself, Ergodic Theory Dynam. Systems, 1986, vol. 6, no. 3, pp. 17-132.

[10] Moreira, P. C. and Gaspar Ruas, A. A., Metric Properties of Cherry Flows, J. Differential Equations, 1992, vol. 97, no. 1, pp. 16-26.

[11] Palmisano, L., A Phase Transition for Circle Maps and Cherry Flows, Commun. Math. Phys., 2013, vol. 321, no. 1, pp. 135-155.

[12] Palmisano, L., On Physical Measures for Cherry Flows, Fund. Math., 2016, vol. 232, no. 2, pp. 167-179.

[13] Palmisano, L., Cherry Flows with Non-Trivial Attractors, Fund. Math., 2019, vol. 244, no. 3, pp. 243-253.

[14] Palmisano, L., Quasi-Symmetric Conjugacy for Circle Maps with a Flat Interval, Ergodic Theory Dynam. Systems, 2019, vol.39, no. 2, pp. 425-445.

[15] Palmisano, L. and Tangue, B., A Phase Transition for Circle Maps with a Flat Spot and Different Critical Exponents, arXiv: 1907.10909 (2019).

[16] Światek, G., Rational Rotation Numbers for Maps of the Circle, Commun. Math. Phys., 1988, vol. 119, no. 1, pp. 109-128.

[17] Tangerman, F. M. and Veerman, J. J.P., Scalings in Circle Maps: 1, Commun. Math. Phys., 1990, vol. 134, no. 1, pp. 89-107.

[18] Tangerman, F. M. and Veerman, J. J.P., Scalings in Circle Maps: 2, Commun. Math. Phys., 1991, vol. 141, no. 3, pp. 279-291.

[19] Veerman, J. J. P., Irrational Rotation Numbers, Nonlinearity, 1989, vol. 3, no. 3, pp. 419-428. 